\title{
QoS Policies for Business Processes in Service Oriented Architectures
}

\author{
Fabien Baligand ${ }^{1,2, \star}$, Nicolas Rivierre ${ }^{1, \star}$, and Thomas Ledoux ${ }^{2}$ \\ ${ }^{1}$ France Telecom - R\&D / MAPS / AMS, \\ 38-40 rue du general Leclerc, 92794 Issy les Moulineaux, France \\ fabien.baligand@gmail.com, nicolas.rivierre@orange-ftgroup.com \\ 2 OBASCO Group, EMN / INRIA, Lina \\ Ecole des Mines de Nantes, \\ 4, rue Alfred Kastler, F - 44307 Nantes cedex 3, France \\ thomas.ledoux@emn.fr
}

\begin{abstract}
The advent of Service Oriented Architectures tends to promote a new kind of software architecture where services, exposing features accessible through highly standardized protocols, are composed in a loose coupling way. In such a context, where services are likely to be replaced or used by a large number of clients, the notion of Quality of Service (QoS), which focuses on the quality of the relationship between a service and its customers, becomes a key challenge. This paper aims to ease QoS management in service compositions through a better separation of concerns. For this purpose, we designed QoSL4BP, a domain-specific language which allows QoS policies specification for business processes. More specifically, the QoSL4BP language is designed to allow an architect to specify QoS constraints and mechanisms over parts of BPEL compositions. This language is executed by our ORQOS platform which cooperates in a non-intrusive way with orchestration engines. At pre-deployment time, ORQOS platform performs service planning depending on services QoS offers and on the QoS requirements in QoSL4BP policies. At runtime, QoSL4BP policies allow to react to QoS variations and to enact QoS management related mechanisms.
\end{abstract}

\section{Introduction}

QoS management in service compositions presents multiple challenges both statically and at runtime. Static time occurs before a composition is deployed on the orchestration engine. This step requires the selection of services whose QoS offers can satisfy the QoS requirements of the composition [18. Because the number of functionally equivalent services is likely to grow larger over the Web, it becomes crucial for architects to have methods and tools allowing them to specify, compute and guarantee QoS of their compositions [7]. Runtime occurs while the composition is executed on the orchestration engine. At this step, QoS

\footnotetext{
* This work was partially supported by the FAROS research project funded by the
} French RNTL. 
of services may vary. Such variations are likely to violate guarantees and lead to variations of the composite service level that need to be dynamically counterbalanced [4. Furthermore, in addition to performance, QoS requirements such as security, reliable messaging and transaction, which rely on WS-* protocols, are major QoS features in service composition that must be addressed [8]. Many solutions offer some interesting methods and tools for QoS management, but we think that none of them takes into account five criteria that seem particularly relevant to the issue of QoS management:

- Reuse of existing standards criterion focuses on the capacity of approaches to leverage standards like BPEL language or Service Level Agreement (SLA) 4]. On the whole, although BPEL is almost always used, few approaches reuse works around SLA.

- Separation of concerns criterion aims to evaluate how service composition and QoS management logics are isolated one from another. Although many approaches promote a better separation of concerns, very few concretely achieve a precise analyze of roles as well as their domains of concern. This often leads to tangled logics or intrusive platforms [16 2], hence reducing maintainability and reusability.

- Coverage of QoS management criterion evaluates how much of QoS domain the solution handles. More specifically, QoS domain includes performance properties [187], such as availability or throughput, as well as non functional properties, like security or reliable messaging 9]. We noted that approaches tackle a wide variety of properties in regard to QoS but few or none actually consider both performance properties and non functional mechanisms (such as security). With emerging works around SLA, taking into account these various properties tends to become necessary for anyone who deals with QoS in business processes.

- Dynamicity criterion shows how approaches can handle QoS management statically, at runtime or both. The study of this criterion tends to show that approaches are fairly dedicated to either static time [7] or runtime [18, hence reducing the scope of applications of these solutions.

- Expressivity criterion evaluates how rich and complex the interfaces offered by the approaches for QoS specification are. Although some approaches include a language to specify QoS management, the expressivity of these languages can be either restrictive [12, or complex to handle [16].

Our approach aims to bring solutions to the challenges of QoS management in SOA by creating a Domain-specific Language (DSL). This language, called "QoSL4BP" (Quality of Service for Business Process), is executed by our platform, namely "ORQOS" (ORchestration Quality Of Service), that performs both at pre-deployment time (for static QoS verification) and at runtime (for dynamic QoS adaptation), as detailed in [1. The remainder of the paper is organized as follows: Section 2 presents the key decisions for the design of our approach. Section 3 presents a scenario from telecommunication world. Section 4 describes the QoSL4BP language, and Section 5 details its execution model. Section 6 discusses the related works. Finally, Section 7 concludes and outlines future work. 


\section{Motivation}

Study of roles. To materialize QoS management and service composition concerns, we identify roles surrounding service compositions, as depicted in Figure 1. Among these roles, the "Composition Architect" role is in charge of the development and of maintaining the functional part of the service composition using BPEL language. The "Integrator Architect" role is in charge of managing the QoS of the service composition by binding the potential Service Providers to the service composition and by managing the relation with each Service Provider. In particular, the Integrator Architect has to negotiate contracts with each Service Provider in order to find appropriate candidates implementing the interfaces provided by the Composition Architect. He can also specify fine-grained requirements on certain parts of the composition and perform QoS related mechanisms like security or replanning strategies on some limited scopes of the composition. For that matter, he requires a "grey box" vision of the service composition, consisting in workflow activities and messages being exchanged. This role is bring to additional QoS requirements to improve QoS or gain profits. For services whose SLA is missing, he can attach QoS offers so that service planning can still be performed. Also, he can specify specific strategies for service planning statically and at runtime, hence reducing the computation cost.

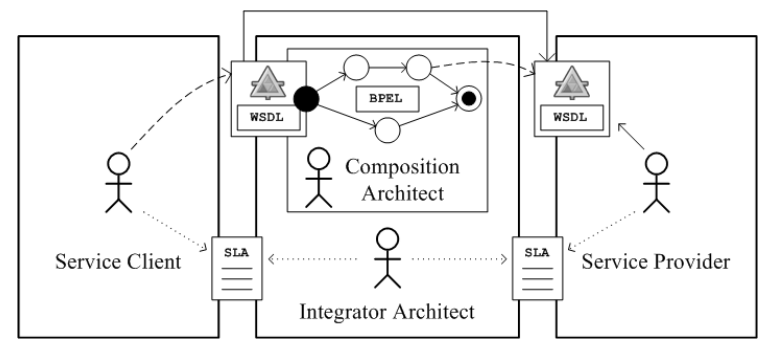

Fig. 1. Roles in service compositions

Domain Study of QoS Management. QoS management requires a variety of treatments and data that characterizes the Integrator Architect role and encompasses the study domain we are interested to capture.

Data related to QoS Management in Business Processes. As depicted in Figure 2, the data related to our domain encapsulates the data belonging to the SLA domain, to the WS-* mechanisms domain, and to the BPEL domain. A SLA specifies the QoS between a client and a provider. In particular, we focussed on the WS-Agreement standard which is composed of terms referencing a party and a SLO acting as an offer guaranteed by the targeted party. A SLO specifies either a performance constraint or WS-* mechanisms. WS-* mechanisms domain refers to the standards described in the Web Service Architecture such as WSSecurity or WS-ReliableMessaging. Finally, BPEL domain is reified into basic or composite BPEL activities which may be linked to partners as well as may contain 


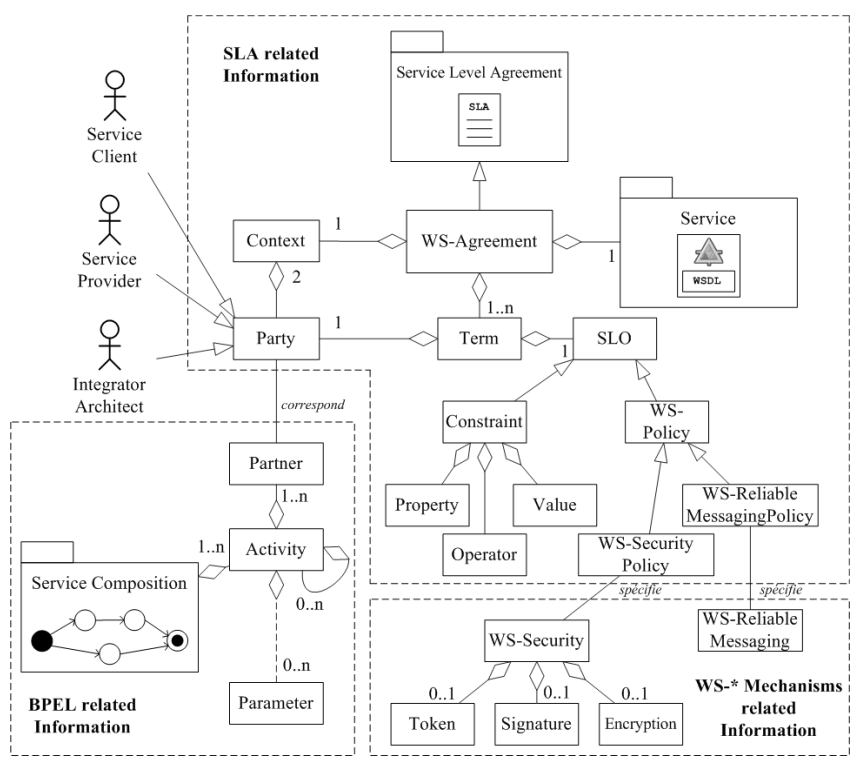

Fig. 2. Data related to QoS Management in Business Processes

additional parameters useful for QoS computation (e.g rate of path selection in Switch activities or the average number of loops in Loop activities).

Treatments related to QoS Management in Business Processes. The Integrator Architect role is bound to a variety of tasks that are described as treatments in our domain study. Such treatments are depicted on Figure 3. First, the Integrator Architect is in charge of SLA management with the partners of the business process (client and Service Providers). This implies selection of QoS offers that guarantee the service level specified in the business processes. The Integrator Architect also deals with QoS observation through QoS monitoring and BPEL parameters supervision. Finally, he manages WS-* mechanisms enactment as described by the WS-* standards.

Domain Specific Language Orientation. To provide the Integrator Architect role with a programmatic interface, we chose to design a Domain Specific Language (DSL) [10] capturing the domain of QoS management in business processes. A DSL is a high-level language providing constructs appropriate to a particular class of problems. Domain expertise is made explicit in the language abstractions and directly supported through its implementation rather than coded by the programmer. The avoidance of low-level source code in itself improves program robustness. More importantly, the use of domain-specific constructs enables or facilitates precise, domain-specific verifications of properties, such as termination properties or critical safety properties, which would be impossible or costly to perform on code written in a general-purpose language. By integrating the concepts of the domain we analyzed, our DSL aims to provide the Integrator Architect role with high level descriptions for QoS management in business processes. 


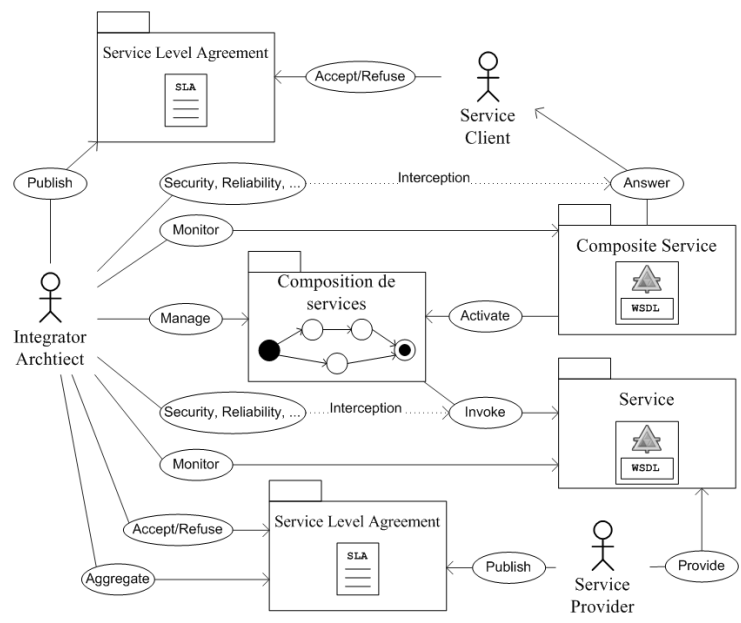

Fig. 3. Activities related to QoS Management in Business Processes

Non Intrusivity. Our contribution lays on the principle of separation of concerns by isolating the logic responsible for QoS management in a DSL from the business process logic. However, the execution of these two logics requires a recomposition method. In order to be able to reuse both the BPEL language as well as the BPEL platforms, we chose not to modify neither the BPEL language nor an existing BPEL platform. Thus, our strategy consists in binding the two languages via loose coupling. Indirections are inserted into the code of the primary concern (i.e BPEL document) in order to call the QoS logic. Such a strategy aims to be non intrusive with existing languages and platforms.

\section{Case Study}

Scenario. Depicted in Figure 4, the "Urban Trip Planner" (UTP) scenario is an exampl 11 of Web Service orchestration, that we used to exemplify our approach. The UTP service aims to plan trips in big cities by delivering the complete transportation route and commutes list as well as a map showing the path from the last station to the final destination. The UTP Service is composed of multiple services. It requires both a destination and a device identification number as inputs. Next, the request is sent to two different services in parallel. These services belong to a flow activity named "LocationScope". The first service uses the device identification number and returns the client current location (for instance, using a Wifi access point location service). The second service takes the destination in input and returns the exact address, using the Yellow Pages

${ }^{1}$ Although being an imaginary use case, this scenario corresponds to issues that companies like France Telecom have to deal with. 


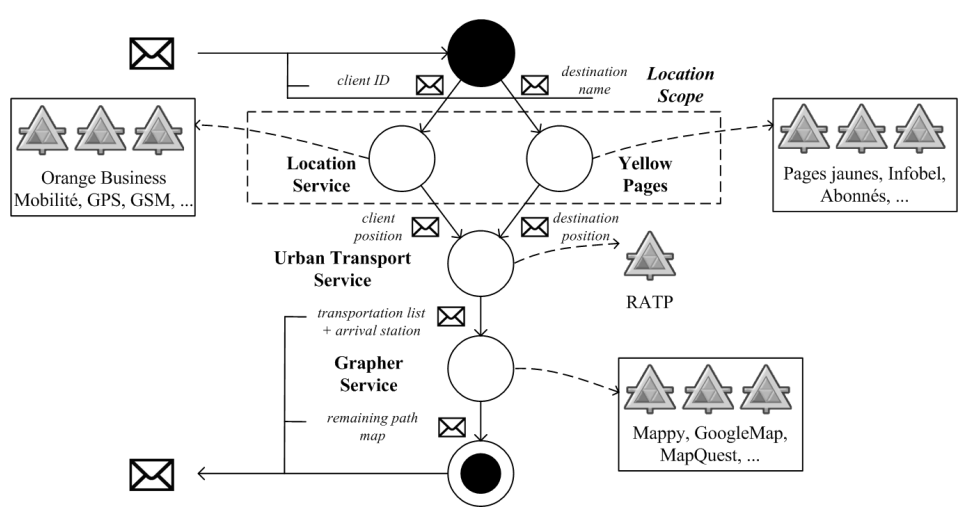

Fig. 4. UTP Service Composition

service. Upon reception of both replies, the UTP service sends both addresses to a Transportation service that returns the route details. The final station address and the destination address are sent to a Grapher service that delivers a map of the path from the station to the destination. Eventually, both the route details and the map are returned to the user.

QoS Requirements. The Integrator Architect may specify multiple requirements related to QoS management. For example: Global QoS Offers. The Integrator Architect wants to guarantee the UTP service QoS to UTP clients. For service selection, the Integrator Architect choose to apply more constrained requirements to make a profit and to ensure that the QoS offers are respected. He also wants to set up a reliable messaging mechanism with the client. If these requirements are not maintained at runtime, replanning must be triggered. Missing SLA. The transportation service is unique (RATP service) and does not provide QoS offers. However, the Integrator Architect found out empirically that its QoS exhibits a response time less than $5 \mathrm{~ms}$, a throughput over 200 requests per minute and a cost equal to 10 cents per call. He has to isolate this service and make up QoS offers. Should the QoS of the service vary, then UTP service should fail. LocationScope Specific Management. In order to reduce the computation task of guaranteeing the global QoS, the Integrator Architect requires that the composite activity "LocationScope" handles its own QoS. In particular, it should handle service planning and replanning. Also, an encryption mechanism is required with the partners of the activities included in "LocationScope" to preserve confidentiality of exchanged messages. Grapher Service Rationing. In order to reduce the selection process for this service, the Integrator Architect asks an additional constraint to set a maximum cost for the Grapher service.

\section{A Language for QoS Policies Specification}

Design. The QoSL4BP language was elaborated, based on our domain study. Thus, QoSL4BP language includes a data model that reifies the information 
associated with this domain, as well as primitives which capture the mechanisms described in this domain. To design the QoSL4BP language, we focus on a couple of properties.

First, QoSL4BP is conceived as a high-level and declarative language. Such a property implies that the programmer manipulates concepts close to the domain idioms and is not required to deal with complex control of algorithms. To ensure that, QoSL4BP enables specification of QoS objectives and its expressivity is limited to a set of relevant mechanisms and data. Thus, QoSL4BP language aims to bring an appropriate balance between rich expressivity and complexity.

Secondly, QoSL4BP language brings a clear separation between static and dynamic specifications by isolating the specifications into two sections. This hybrid approach helps bringing more dynamicity in order to handle QoS management effectively both at pre-deployment time and at runtime. QoSL4BP language provides support for QoS management at diverse granularity levels (whole business process, basic or composite activities), which helps to rationalize QoS management. To increase homogeneity and reusability, the QoSL4BP specifications are contained in modules called "policies". Finally, QoSL4BP language possesses some guarantees relevant to QoS management in business processes. For brevity's sake, we do not provide details about them in this paper. However, these guarantees ensure BPEL and QoSL4BP logics synchronisation, BPEL activity types verification (basic or composite activities), policy composition (when two policies interact, specifications of the policy whose target granularity is the finest override those of the policy whose rather granularity is coarser), exception handling and termination.

Structure. The structure of QoSL4BP policies is shown in Figure 5. They are made of three sections: the section SCOPE describes the BPEL activities target whose QoS management logic is attached to, whereas the sections INIT and RULES respectively contain the static and dynamic logics specification. Thus, INIT specifications are executed at pre-deployment time and RULES specifications are executed at runtime. Instructions of the section RULES can be written as "condition-action" rules.

The data model of QoSL4BP language emerges from the domain study, depicted in Figure 2, Based on this study, we choose BPEL activities and SLA information as structuring elements for the data model of QoSL4BP language. BPEL activities are used both to specify the SCOPE section of the policy and to designate the target instructions in the INIT and RULES sections. In particular, in QoSL4BP, a BPEL activity may be linked to one or several partners,

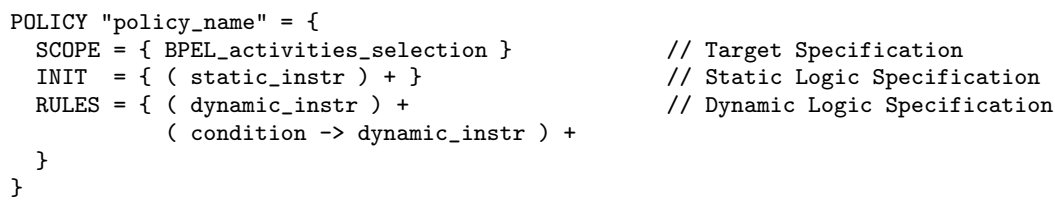

Fig. 5. QoSL4BP Policy Structure 
hence allowing to easily attach some QoS offers, requirements or mechanisms. To enable the programmer to efficiently point out BPEL activities in a business process, QoSL4BP offers a specific semantic including some activity composition operators. SLA information enables to specify QoS data related to QoS performance or mechanisms. In QoSL4BP, a SLA consists in a collection of terms. A term is made of one QoS constraint and of a party responsible for the QoS constraint. A QoS constraint can considered either as an offer or as a requirement depending if the constraint is guaranteed to an external party or if an external asks for the constraint to be applied. Constraints can be specialized either into a performance constraint (e.g Response time less than $10 \mathrm{~ms}$ ) or into a QoS mechanism (e.g encryption using the "RSA algorithm").

Similarly to the data model, the primitives emerge from the domain study and aim to provide the Integrator Architect with expressivity to perform the activities depicted in Figure [3, Primitives appear in Figure 6] and are categorized depending on their concern and on the parties involved: The primitives SET_OFFER and SET_REQUIREMENT makes it possible to set QoS constraints which are processed at service selection, performed using primitive PLANNING. Services can be manually set or obtained using the BIND and BOUND primitives. READ_CLIENT and READ_PROVIDER allow to read constraints from accepted SLA. The primitives SENSOR and MONITOR return monitored QoS values at runtime. VIOLATION_ACTIVITY and VIOLATION_PROVIDER allows to verify whether the requirements of a SLA are not violated. The primitives PERFORM_CLIENT and PERFORM_ACTIVITY allow to specify a WS-* mechanism to perform either with the client, or with one to several activities. THROW and CATCH are means to manage exceptions.

Illustrations with UTP Scenario. For brevity's sake, we exhibit only two out of the four policies. Figure 7 exhibits the policy "selection_RATP" corresponding to the QoS requirement called Missing SLA. This policy applies to the "InvokeTransportation" activity. At pre-deployment time, some QoS offers are attached to this activity and the service is bound to the partnerlink "WS_RATP". At

\begin{tabular}{|c|c|c|c|c|}
\cline { 2 - 5 } \multicolumn{1}{c|}{} & Integrator-Client & Integrator-Activity & Integrator-Provider \\
\hline \multirow{4}{*}{ SLA Management } & Constraints & SET_OFFER & $\begin{array}{c}\text { SET_OFFER } \\
\text { SET_REQUTREMENT }\end{array}$ & SET_REQUREMENT \\
\cline { 2 - 5 } & Planning & & PLANNING & $\begin{array}{c}\text { PLANNING } \\
\text { BIND } \\
\text { BOUND }\end{array}$ \\
\cline { 2 - 5 } & Access & READ_CLIENT & READ_PROVIDER \\
\hline \multirow{4}{*}{ QoS Observation } & Monitoring & SENSOR & $\begin{array}{c}\text { SENSOR } \\
\text { MONITOR } \\
\text { QEST/GET_RATE } \\
\text { SET/GET_LOOP }\end{array}$ & MONITOR \\
\cline { 2 - 6 } & Violation & VIOLATION_ACTIVITY & VIOLATION_ACTIVITY & VIOLATION_PROVIDER \\
\cline { 2 - 5 } & WS-* & PERFORM_CLIENT & PERFORM_ACTIVITY & PERFORM_ACTIVITY \\
\cline { 2 - 5 } & Exception & THROW & $\begin{array}{c}\text { CATCH } \\
\text { (LOG) }\end{array}$ & \\
\hline
\end{tabular}

Fig. 6. QoSL4BP Primitives 


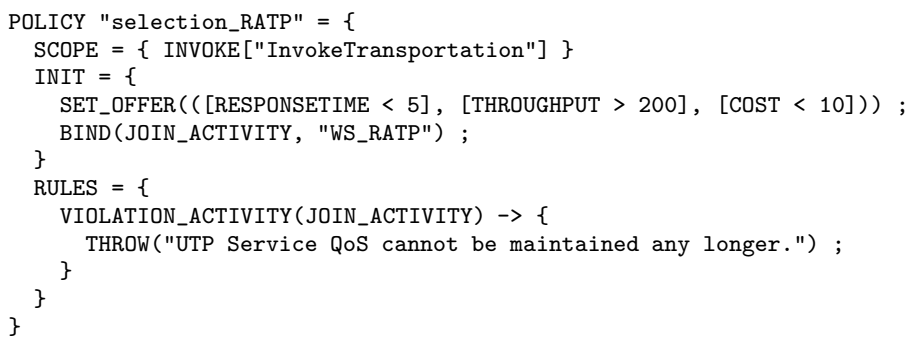

Fig. 7. "selection_RATP" Policy

runtime, if the QoS requirements for the "LocationScope" activity are violated, then the UTP service should send an exception.

Figure 8 exhibits the policy "location_security" corresponding to the QoS requirement LocationScope Specific Management. This policy applies to the "LocationScope" flow activity. At pre-deployment time, some QoS offers and requirements are attached to this activity. Service planning is performed on the target activity using a constraint programming algorithm (CP) [1]. At runtime, two encryption mechanisms are performed for the invoke activities of "LocationScope". Should a security exception be raised, then it should be logged and the UTP service should send an exception. Also, if the QoS requirements for the "LocationScope" activity are violated, then service replanning is to be performed. Should this replanning fail, then it should be logged and the UTP service should send an exception.

\section{Execution Model}

Global process. Figure 9 depicts the global process of QoSL4BP policies execution, including three steps. Before this process takes place, the Composition Architect must provide a BPEL document and a set of generic WSDL describing the functional interface of each service involved in the composition. The Integrator Architect specifies QoSL4BP policies and a list of Service Providers for each service of the composition.

(1) First, the static specifications (from the INIT section) are executed by ORQOS. The values are set for the initial states of the system (offers and requirements of activities, BPEL parameters). At this step, ORQOS performs service planning to find a set of initial Service Providers. This results either in a failure or a successful attempt for service planning. (2) The second step also occurs before the deployment of the BPEL composition. It aims to modify the BPEL document by weaving indirections (or "hooks") calling ORQOS platform at specific points requiring QoS policies execution. (3) Final step occurs at runtime, when the composition is executed by clients and calls Service Providers. At this step, ORQOS platform is called by the hooks from the BPEL document. It also intercepts messages between the composition and its partners to perform the appropriate treatments (monitoring, security, service replanning, etc.). 


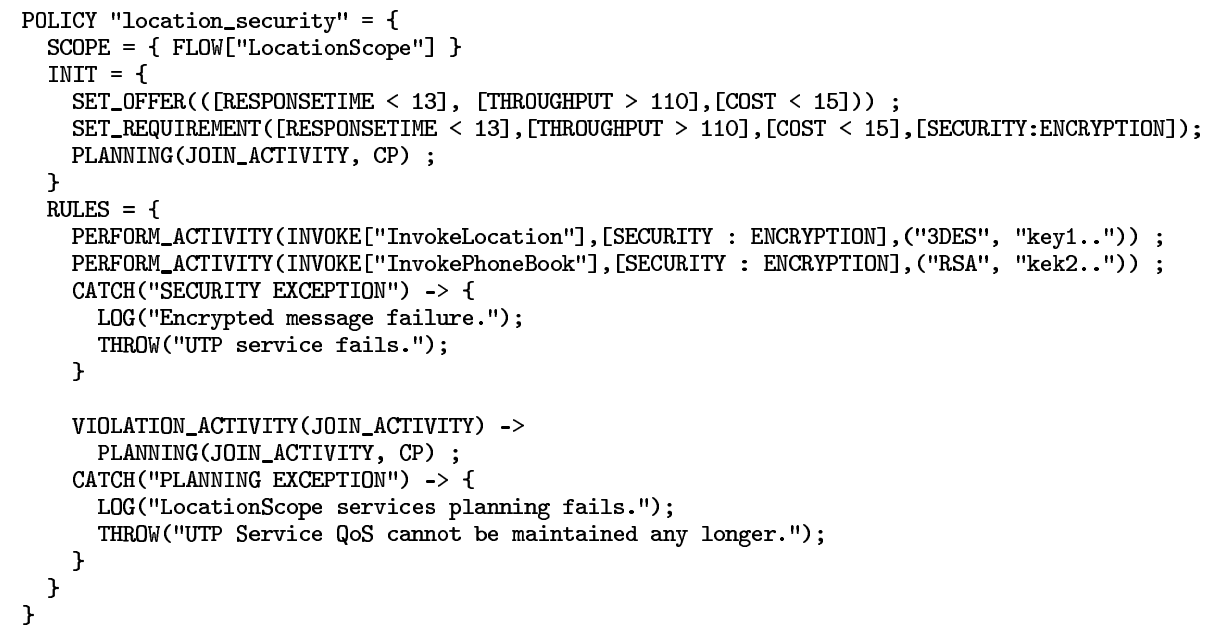

Fig. 8. "location_security" Policy
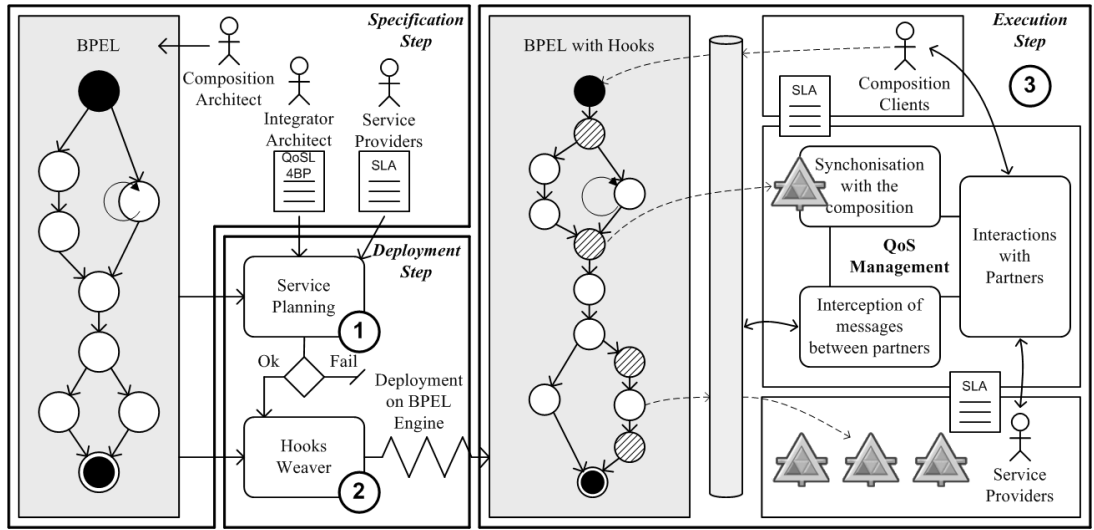

Fig. 9. Global Process

Static planning. First, the BPEL composition is translated into a tree, as shown in Figure 10. Composite activities are translated into nodes whereas basic activities become leaves. The values specified in the INIT section of policies (QoS offers, requirements, etc.) are attached to the elements of this tree. Using a tree as a model to reflect on QoS of the composition allows to efficiently associate QoS information and structure of this information.

Next, the tree is decomposed into multiple sub trees: for each element containing QoS offers, this element and its potential leaves are detached to become a new sub tree. This transformation is also depicted in Figure 10, Such decomposition allows to reduce the planning computation effort due to the fact that activities containing QoS offers act as guarantees to the rest of the composition. 
Thus, the maximal computational complexity is $\sum_{i=0}^{n} s^{c_{i}}$ which is in $\Theta\left(s^{\max \left(c_{i}\right)}\right)$ (with $n$ the number of sub trees, $c_{i}, i \epsilon[1, n]$ the number of leaves in the sub tree $i$, and $s$ the average number of Service Providers).

Finally, service planning is performed in each sub tree using QoS offers and requirements, SLA data of each Service Provider, as well as composition rules as described in [5]. Although ORQOS may implement any existing algorithm, it offers so far two algorithms, using constraint programming and backtracking. Constraint programming is performed by translated each sub tree into a constraint network then by using a constraint solver tool. Backtracking algorithm is performed by exhaustively trying each SLA offer of Service Provider and testing against QoS requirements from the bottom to the top of each sub tree, back and forth when it fails.

Preparation of the composition. At pre-deployment time, some hooks are weaved before and after each BPEL activity. These hooks consist in invoke activities calling an ORQOS platform Web service interface. This enables to execute policies without modifying the BPEL engine. Next, in order to perform some mechanisms (e.g monitoring or security), ORQOS platform requires to intercept the messages exchanged between the composition and its partners. For this purpose, ORQOS platform acts as a proxy. In particular, partnerlinks of the BPEL document are redirected to the ORQOS platform and includes header identification information so that ORQOS can bind received messages with their activities.

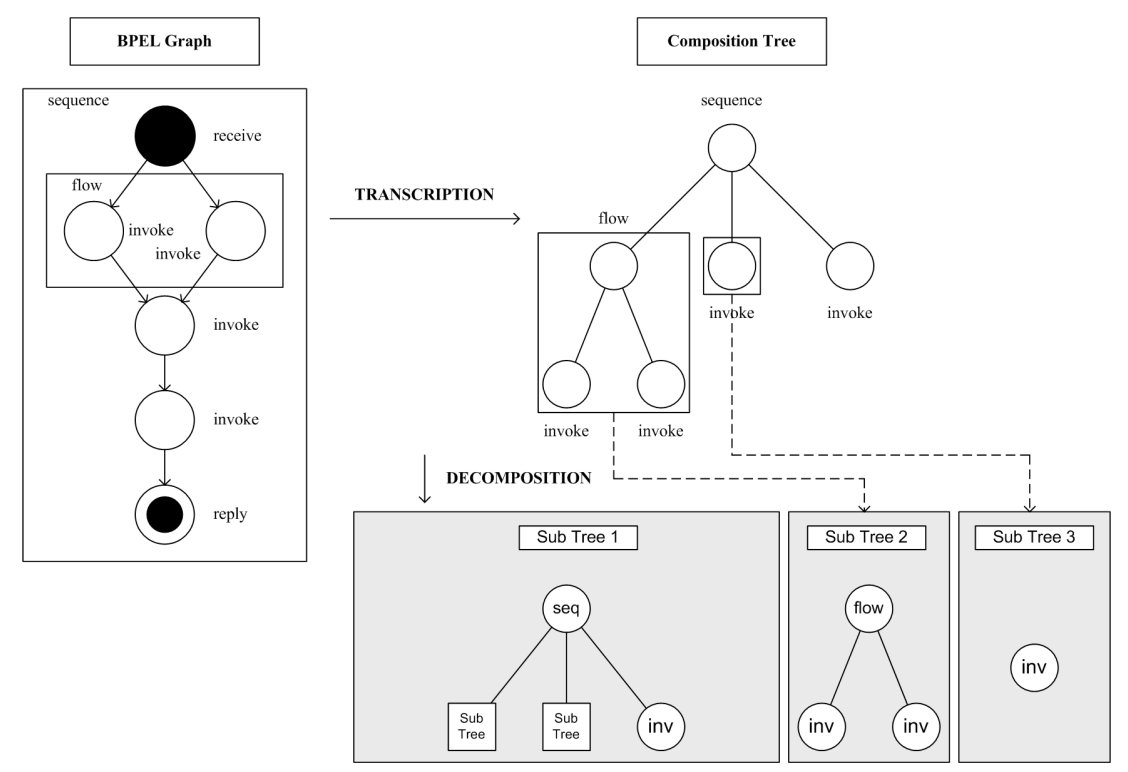

Fig. 10. Transcription and Decomposition 
Dynamic Execution. The execution of QoSL4BP policies processes the RULES sections of the policies at runtime. Synchronization between BPEL and policies is achieved by the hooks weaved at pre-deployment time. Rules are executed before and after each activity since the QoS state of the system is likely to change after the execution of the targeted activity, called "Join Activity" similarly to "Join Point" in Aspect Oriented Programming [13]. To perform the mechanisms specified in the rules, ORQOS platform integrates already existing components such as Apache WSS4J, Sandesha and WSAG4J which implements WS-Security, WS-ReliableMessaging and WS-Agreement SLA management logics respectively.

\section{Related Works}

Reuse of existing standards. Except AgFlow 17/18 and WS-Binder [144, few approaches reuse SLA works to handle QoS in compositions. In [18 the authors tackle the issue of replanning as a graph search problem, using integer programming technique for service selection. Their method allows to select, for each abstract service of a workflow, a concrete service so that their QoS aggregation is optimized and that the global constraints are satisfied. WS-Binder performs service planning, and it allows to specify and to compose domain specific QoS properties. Our approach reuses both SLA semantic (QoS offers and requirements integrated in QoSL4BP language) and tools (WS-Agreement protocol implementation). It provides the Integrator Architect with flexibility to decide about the triggering decision and about the BPEL activities where service replanning should occur.

Separation of concerns. Each platform that handles QoS in service compositions has to deal with QoS management concern and business process concern collaboration. Some approaches, such as DYNAMO [2]3 and MASC [16, focus on how to isolate the specifications of these logics. DYNAMO provides two languages, WSCoL (Web Services Constraint Language) and WSReL (Web Service Recovery Language), that aim to allow the specification of monitoring and reactions in BPEL processes. In MASC (Manageable and Adaptive Service Compositions), the authors bring an Event-Condition-Action based language called WS-Policy4MASC allowing to specify adaptation strategies in service compositions. However, the tools in charge of the execution of these specifications are tangled up which prevent from reusing BPEL engines without modification. Other works, like TRAP/BPEL [12] or WS-Binder, isolate both the specifications and the execution tools. TRAP/BPEL is based on Transparent shaping [15] which aims to bring new behaviors in a non intrusive way by inserting hooks calling the logic of these behaviors at runtime. Our approach is based on a similar strategy that allows both specifications (BPEL and QoSL4BP) and implementation platforms (BPEL engine and ORQOS platform) to be kept isolated. This strategy increases logic and platform maintenance, evolutivity and reusability. 
Furthermore, because separation of concerns is a major focus in our work, we created the Integrator Architect role and emphasized its domain of concern.

Coverage of QoS management. On the whole, platforms focus either on performance properties concerns (AgFlow and ORBWork [76]) either on non functional concerns such as security or reliable messaging (AO4BPEL 89 9]). ORBWork propose mathematical models for workflow QoS computation to predict QoS, using an algorithm consisting of a set of graph reduction rules which are defined for time, cost, reliability and fidelity metrics. In AO4BPEL, the authors have elaborated a language named "Aspect Oriented for Business Process Execution Language" (AO4BPEL) to bring AOP mechanisms to the BPEL language. By using the SLA as a fundamental basis for our language, our approach aims to provides answer to any QoS concern that can be described by a Service Level Objective (either performance properties or WS-* mechanisms). Also, because policies are attached to BPEL activities, this enables to apply QoS management to various granularity levels (whole composition, set of activities, basic activity).

Dynamicity. Many approaches (WS-Binder, AO4BPEL, DYNAMO) perform QoS management only during the execution of the business processes and do not consider QoS at pre-deployment time. However, ORBWork brings QoS guarantees or perform service planning at pre-deployment time but do not consider QoS at runtime. Also, AgFlow and WS-Binder platforms offer static solutions for service planning at pre-deployment time, as well as strategies for adaptation at runtime. By handling both predeployment time and runtime for QoS management, our approach aims to provide the Integrator Architect with abilities to compute global service planning statically and to specify fine-grained strategies for dynamic service replanning.

Expressivity. Solutions like AO4BPEL, DYNAMO or MASC provide languages, while some others, such as WS-Binder or AgFlow, have the QoS management logic directly integrated in their platforms. DYNAMO offers to manipulate supervision and recovery with its two languages in a declarative mode. MASC provides a language that aims to answer to a wide variety of concerns, which introduces complexity. AO4BPEL reuses AspectJ syntax, XPath and BPEL language to allow the user to specify BPEL adaptation aspect in a imperative mode. To provide a relevant expressivity and to encapsulate efficiently QoS management concerns, QoSL4BP language was designed as a declarative language based on a precise study of the domain of QoS management in business processes. In particular, it enables to specify using SLA and BPEL activities information, and its expressivity is limited to a reasonable amount of primitives, making it efficient to use yet relatively easy to learn.

\section{Conclusion}

In this paper, we presented a language and a platform addressing QoS management in business processes, both at predeployment time and at runtime. Our language called "QoSL4BP" (Quality of Service Language for Business Processes) allows an architect to specify QoS policies over scopes of BPEL compositions. 
Static and dynamic execution of the QoSL4BP language are performed by our platform "ORQOS" (ORchestration Quality of Service), designed to be non intrusive with already existing infrastructures and languages. At pre-deployment time, ORQOS selects a set of services whose QoS offers match the QoS requirements attached to the BPEL activities of the composition. At runtime, ORQOS platform monitors the QoS of services and performs actions such as selecting different Service Providers, throwing BPEL exceptions, or performing WS-* mechanisms, as specified in QoSL4BP policies.

Although solutions to deal with QoS management in business processes already exist, our approach aims to answer to some issues often encountered by these solutions. First, it focuses on reusing most of the already existing SOA standards such as BPEL, SLA and WS-* norms. It aims to provide a better separation of concern by achieving a precise study of the domain of QoS management and by encapsulating this domain in QoSL4BP language as well as in ORQOS platform (which is non intrusive with BPEL engines). Our approach tends to allow the architect to manipulate both QoS performance properties and QoS non functional properties such as security, at pre-deployment and at runtime. Finally, QoSL4BP is designed as a declarative policy-based language that allows to manipulate concepts close to the domain idioms without being required to deal with complex control of algorithms.

In future works, we plan to enhance QoSL4BP language by addressing a larger number of QoS dimensions and mechanisms, and by studying the issue of policy composition that occurs when at least two policies scopes intersect. We also plan to investigate formal semantics of QoSL4BP language, as well as its re-design using WS-Policy standard.

\section{References}

1. Baligand, F.: PhD thesis, Une Approche Déclarative pour la Gestion de la Qualité de Service dans les Compositions de Services (June 2008)

2. Baresi, L., Guinea, S.: Dynamo and self-healing bpel compositions. In: ICSE COMPANION 2007: Companion to the proceedings of the 29th International Conference on Software Engineering, pp. 69-70. IEEE Computer Society, Washington (2007)

3. Baresi, L., Guinea, S., Plebani, P.: Ws-policy for service monitoring. In: Bussler, C.J., Shan, M.-C. (eds.) TES 2005. LNCS, vol. 3811, pp. 72-83. Springer, Heidelberg (2006)

4. Canfora, G., Di Penta, M., Esposito, R., Perfetto, F., Villani, M.L.: Service composition (re)binding driven by application-specific qos. In: Dan, A., Lamersdorf, W. (eds.) ICSOC 2006. LNCS, vol. 4294, pp. 141-152. Springer, Heidelberg (2006)

5. Cardoso, J., Miller, J., Sheth, A., Arnold, J.: Modeling quality of service for workflows and web service processes (2002)

6. Cardoso, J., Miller, J., Sheth, A., Arnold, J.: Modeling quality of service for workflows and web service processes. Technical Report UGACS-TR-02-002, Computer Science Department, University of Georgia (2002)

7. Cardoso, J., Sheth, A., Miller, J., Arnold, J., Kochut, K.: Quality of service for workflows and web service processes. Web Semantics: Science, Services and Agents on the World Wide Web 1(3), 281-308 (2004) 
8. Charfi, A., Mezini, M.: Aspect-oriented web service composition with AO4BPEL. In: Zhang, L.-J., Jeckle, M. (eds.) ECOWS 2004. LNCS, vol. 3250, pp. 168-182. Springer, Heidelberg (2004)

9. Charfi, A., Schmeling, B., Heizenreder, A., Mezini, M.: Reliable, secure, and transacted web service compositions with ao4bpel. In: Proceedings of the 4th IEEE European Conference on Web Services (ECOWS) (December 2006)

10. Consel, C.: Charles Consel. In: Lengauer, C., Batory, D., Consel, C., Odersky, M. (eds.) Domain-Specific Program Generation. LNCS, vol. 3016, pp. 19-29. Springer, Heidelberg (2004)

11. Dechter, R.: Constraint Processing. Morgan Kaufmann Publishers, San Francisco (2003)

12. Ezenwoye, O., Sadjadi, S.M.: Trap/bpel: A framework for dynamic adaptation of composite services. Technical Report FIU-SCIS-2006-06-02 (2006)

13. Kiczales, G., Lamping, J., Menhdhekar, A., Maeda, C., Lopes, C., Loingtier, J.M., Irwin, J.: Aspect-oriented programming. In: Aksit, M., Matsuoka, S. (eds.) ECOOP 1997. LNCS, vol. 1241, pp. 220-242. Springer, Heidelberg (1997)

14. Penta, M.D., Esposito, R., Villani, M.L., Codato, R., Colombo, M., Nitto, E.D.: Ws binder: a framework to enable dynamic binding of composite web services. In: SOSE 2006: Proceedings of the 2006 international workshop on Service-oriented software engineering, pp. 74-80. ACM, New York (2006)

15. Sadjadi, S.M., McKinley, P.K., Cheng, B.H.C., Stirewalt, R.E.K.: Trap/j: Transparent generation of adaptable java programs. In: Meersman, R., Tari, Z. (eds.) OTM 2004. LNCS, vol. 3291, pp. 1243-1261. Springer, Heidelberg (2004)

16. Tosic, V., Erradi, A., Maheshwari, P.: Ws-policy4masc - a ws-policy extension used in the masc middleware. In: IEEE SCC, pp. 458-465. IEEE Computer Society, Los Alamitos (2007)

17. Zeng, L., Benatallah, B., Dumas, M., Kalagnanam, J., Sheng, Q.Z.: Quality driven web services composition. In: WWW 2003: Proceedings of the 12th international conference on World Wide Web, pp. 411-421. ACM, New York (2003)

18. Zeng, L., Benatallah, B., Ngu, A.H.H., Dumas, M., Kalagnanam, J., Chang, H.: Qos-aware middleware for web services composition. IEEE Trans. Softw. Eng. 30(5), 311-327 (2004) 